Research Paper

\title{
Photosensitizer-Conjugated Gold Nanorods for Enzyme-Activatable Fluo- rescence Imaging and Photodynamic Therapy
}

\author{
Boseung Jang and Yongdoo Choi ${ }^{\bowtie}$ \\ Molecular Imaging \& Therapy Branch, Division of Convergence Technology, National Cancer Center, 111 Jungbalsan-ro, \\ Ilsandong-gu, Goyang, Gyeonggi-do 410-769, Republic of Korea
}

$\triangle$ Corresponding author: Yongdoo Choi, PhD, Molecular Imaging \& Therapy Branch, Division of Convergence Technology, National Cancer Center, 111 Jungbalsan-ro, Ilsandong-gu, Goyang, Gyeonggi-do 410-769, Republic of Korea. Tel/Fax: +82-31-920-2512. E-mail: ydchoi@ncc.re.kr

(C) Ivyspring International Publisher. This is an open-access article distributed under the terms of the Creative Commons License (http://creativecommons.org/ licenses/by-nc-nd/3.0/). Reproduction is permitted for personal, noncommercial use, provided that the article is in whole, unmodified, and properly cited.

Received: 2011.09.05; Accepted: 2011.10.07; Published: 2012.02.11

\begin{abstract}
We report on the development of photosensitizer-conjugated gold nanorods (MMP2P-GNR) in which photosensitizers were conjugated onto the surface of gold nanorods (GNR) via a protease-cleavable peptide linker. We hypothesized that fluorescence and phototoxicity of the conjugated photosensitizers would be suppressed in their native state, becoming activated only after cleavage by the target protease matrix metalloprotease-2 (MMP2). Quantitative analysis of the fluorescence and singlet oxygen generation (SOG) demonstrated that the MMP2P-GNR conjugate emitted fluorescence intensity corresponding to $0.4 \% \pm 0.01 \%$ and an SOG efficiency of $0.89 \% \pm 1.04 \%$ compared to free pyropheophorbide-a. From the in vitro cell studies using HTI080 cells that overexpress MMP2 and BT20 cells that lack MMP2, we observed that fluorescence and SOG was mediated by the presence or absence of MMP2 in these cell lines. This novel activatable photosensitizing system may be useful for protease-mediated fluorescence imaging and subsequent photodynamic therapy for various cancers.
\end{abstract}

Key words: Photodynamic therapy, Gold nanorod, Resonance energy transfer, Quenching, Enzyme activatable

\section{Introduction}

Photodynamic therapy (PDT) describes the use of chemical photosensitizers combined with specific wavelengths of light. This form of therapy has been used successfully to treat cancers and is capable of overcoming side effects associated with conventional cancer treatments such as surgery, radiation therapy, and chemotherapy [1-2]. Photosensitizers accumulate in tumor tissues and are typically nontoxic to cells in the absence of light. When photosensitizers are excited by light radiation of a specific wavelength, they react with the surrounding substrates or with molecular oxygen to produce reactive oxygen species (ROS), which selectively damage tumor tissues in situ. However, current photosensitizers can also nonspecifically accumulate in the skin or the eyes of patients. If, following PDT, these patients are exposed to light, such as sunlight or bright indoor light, side effects caused by damage to normal cells in these tissues can occur. Limited tumor selectivity and unfavorable biodistribution of conventional PDT agents have remained major obstacles to their clinical applications. "Activatable PDT" agents have recently attracted a great deal of attention as a strategy to overcome nonspecific activation of phototoxicity. In an ideal 
PDT agent, generation of both fluorescence signal and cytotoxic singlet oxygen would be completely inhibited in its native state, even upon light exposure. Their optical properties would be selectively activated by stimuli such as enzymes [3-6] and pH [7-8]. Choi et al. reported the first successful in vivo results demonstrating the potential use of an activatable PDT agent in near-infrared fluorescence imaging and subsequent PDT of cancers. [3] Various mechanisms for controlling optical properties of photosensitizers have been tested, including self-quenching between the same type of photosensitizers $[3,5-6,9]$, quenching by organic quenchers $[4,10]$, and quenching by carbon nanotubes [11] and gold nanorods [12].

Herein, we report on photosensitizer-conjugated gold nanorods (Figure 1), in which photosensitizers were conjugated onto the surface of gold nanorods
(GNRs) via a protease-cleavable peptide linker. GNRs have immense absorption coefficients, 104- to 106-fold higher than those of conventional organic dyes, and therefore, may serve as ultraefficient energy quenchers of excited photosensitizers through their surface-energy-transfer properties [13-14]. In addition, gold nanoparticles, including GNRs, can quench the excited energy of fluorochromes even at a distance of $\sim 40 \mathrm{~nm}$ [15]. Therefore, we expected the fluorescence and phototoxicity of the photosensitizers conjugated on the surface of GNRs to be suppressed in their native state, and activated following release from the GNR surface by the action of a target protease. This strategy may allow a photodynamic reaction to take place in a target area with greater selectivity and efficiency.

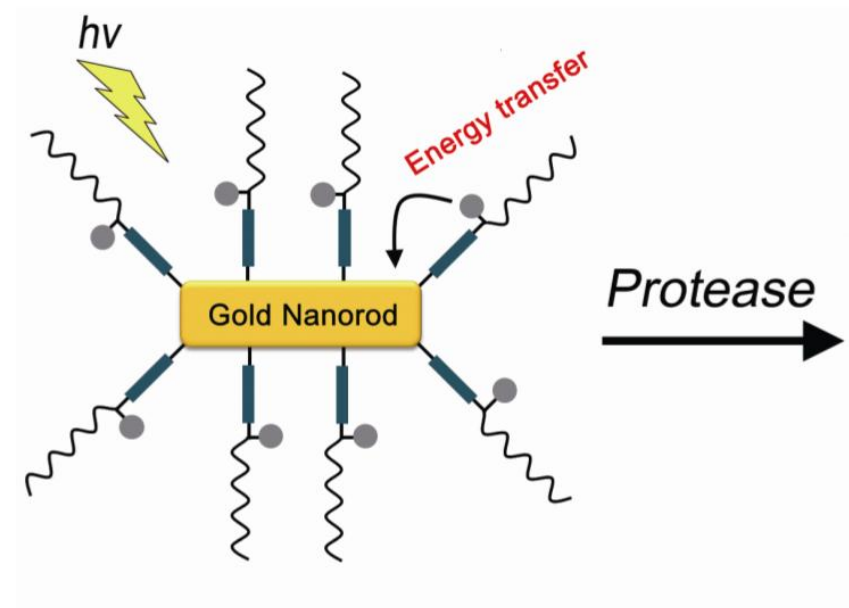

Non-fluorescent Non-phototoxic
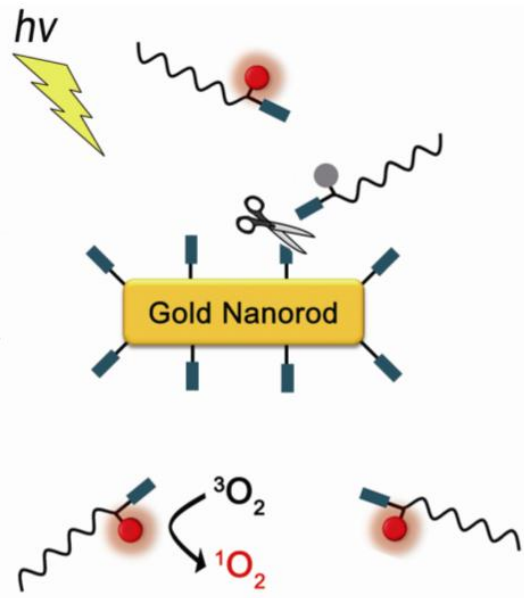

Highly fluorescent Phototoxic

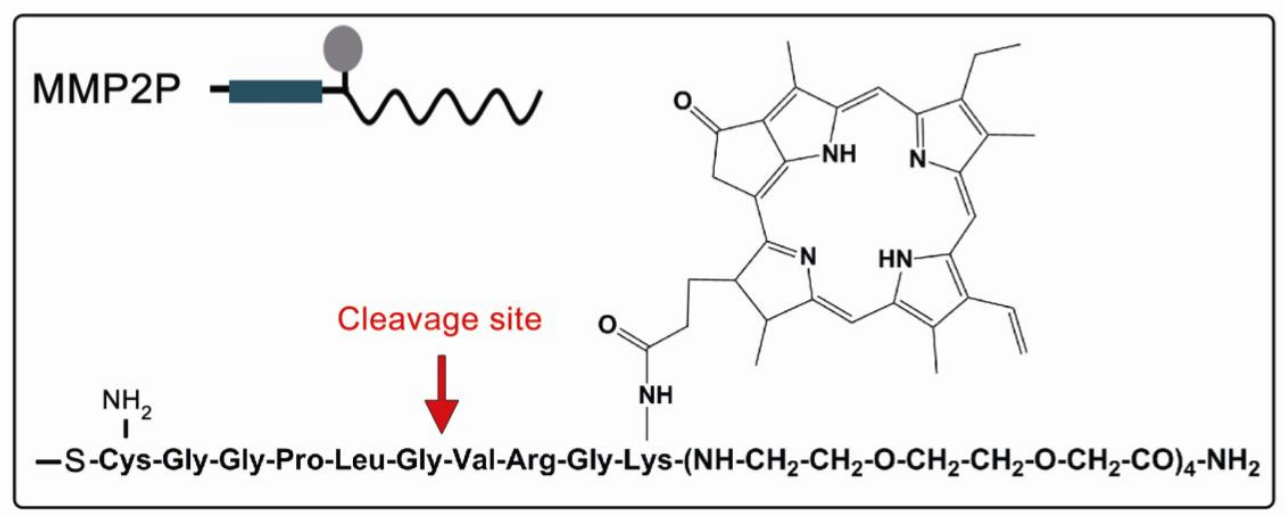

Figure I. A concept of enzyme activatable fluorescence imaging and photodynamic therapy using a photosensitizer-conjugated gold nanorod (MMP2P-GNR). Box: Structure of the MMP2P containing MMP2-cleavable peptide sequence and photosensitizer. Arrow indicates cleavable site by MMP2 activity. 
In this study, the sequence GPLGVRG (-Gly-Pro-Leu-Gly-Val-Arg-Gly-), which is specific to the matrix metalloproteinase-2 (MMP2) substrate (Figure 1), was introduced as the target for enzymatic hydrolysis by MMP2 [16]. Matrix metalloproteinases (MMPs) are zinc-dependent endopeptidases and are known to play key roles in tumor growth, progression, and metastasis as well as in dysregulated angiogenesis [17-20]. Therefore, MMPs, including MMP2, are considered important therapeutic and diagnostic targets for the treatment and detection of human cancers. Cysteine was added to the peptide sequence in order to conjugate MMP2P on the surface of the GNRs via thiol chemistry.

\section{Materials and methods}

\section{Materials}

Gold (III) chloride trihydrate $\left(\mathrm{HAuCl}_{4} \cdot 3 \mathrm{H}_{2} \mathrm{O}\right.$, 99.9\%), sodium borohydride $\left(\mathrm{NaBH}_{4}, 99 \%\right)$, silver nitrate $\left(\mathrm{AgNO}_{3}, 99 \%\right)$ and L-ascorbic acid (AA, 99\%), and hexadecyltrimethylammonium bromide (CTAB, 99\%) were purchased from Sigma-Aldrich (St. Louis, $\mathrm{MO})$. Pyropheophorbide-a (PPa) was purchased from Frontier Scientific, Inc. (Logan, Utah). Singlet oxygen sensor green was purchased from Molecular Probes. Ultrapure deionized water was used in all experiments. HT1080 cells (human fibrosarcoma cell line) overexpressing MMP2 and BT20 cells (human mammary adenocarcinoma cell line), which lack detectable MMP2 expression [16], were obtained from American Type Culture Collection (ATCC, Rockville, MD). Cell lines were maintained in Dulbecco's modified Eagle's medium (DMEM) supplemented with 10\% fetal bovine serum (FBS), (GIBCO, Invitrogen) in a humidified $5 \% \mathrm{CO}_{2}$ incubator at $37^{\circ} \mathrm{C}$.

\section{Preparation of gold nanorod solution}

Gold nanorods were prepared using a seed-mediated method as described previously [12, 21]. In brief, CTAB was dissolved in deionized water (DW) to a molarity of $100 \mathrm{mM} ; 7.5 \mathrm{ml}$ of this CTAB aqueous solution was mixed with $250 \mu \mathrm{L}$ of $10 \mathrm{mM}$ $\mathrm{HAuCl}_{4}$ aqueous solution, followed by the addition of $600 \mu \mathrm{L}$ of $10 \mathrm{mM} \mathrm{NaBH}_{4}$ aqueous solution. The mixture was stirred for $2 \mathrm{~min}$, and maintained at $25^{\circ} \mathrm{C}$ for $2 \mathrm{~h}$ to prepare a seed solution.

Meanwhile, $1.7 \mathrm{~mL}$ of $10 \mathrm{mM} \mathrm{HAuCl}_{4}$ aqueous solution was added to $40 \mathrm{~mL}$ of $100 \mathrm{mM} \mathrm{CTAB}$ aqueous solution, followed by the sequential addition of $250 \mu \mathrm{L}$ of $10 \mathrm{mM} \mathrm{AgNO}_{3}$ aqueous solution and $270 \mu \mathrm{L}$ of $100 \mathrm{mM}$ ascorbic acid. At this point, $420 \mu \mathrm{L}$ of the seed solution was added, and then maintained to react for $12 \mathrm{~h}$. Thereafter, the reaction mixture was cen- trifuged at $15000 \times g$ at $25^{\circ} \mathrm{C}$ for $15 \mathrm{~min}$, thereby obtaining a CTAB-coated gold nanorod.

\section{Preparation of photosensitizer-peptide conju- gate (MMP2P)}

MMP2-cleavable peptide substrate coupled with PPa (MMP2P) was purchased from Peptron Inc. (Daejeon, Republic of Korea). Supplementary Material: Figure S1 briefly summarizes the procedure of MMP2P synthesis. An HPLC and mass analyses of MMP2P shows a purity of about $98 \%$, and a molecular weight of 2039 g/mol (Supplementary Material: Figure S2 and S3).

\section{Preparation of MMP2P-gold nanorod (MMP2P-GNR) conjugate}

The centrifuged CTAB-coated GNRs were resuspended in $1 \mathrm{~mL}$ of DW to remove excess CTAB. The concentration of the GNR solution was $100 \mathrm{nM}$ after resuspension. An aqueous solution was prepared by dissolving the MMP2P to a concentration of $1 \mathrm{mM}$, of which $200 \mu \mathrm{L}$ was added to the dispersion in addition to $100 \mu \mathrm{L}$ of $2 \mathrm{mM} \mathrm{K}_{2} \mathrm{CO}_{3}$ aqueous solution and allowed to react at room temperature for 5 days. The resulting solution was dialyzed in DW using an ultrafiltration membrane with a molecular weight cut-off (MWCO) of 50,000 Da to remove MMP2P that did not participate in the reaction with the GNRs. The remaining solution represented MMP2P-GNR conjugates in which the photosensitizer-oligopeptide combination was linked with the GNRs.

To calculate the number of conjugated MMP2P per GNR, the absorption spectrum of the purified MMP2P-GNR was measured in both DW (Figure 2B) and dimethylformamide (DMF) using a UV/Vis scanning spectrophotometer (DU730, Beckman). The CTAB-coated GNR has a molar absorption coefficient of $4.6 \times 10^{9} \mathrm{M}^{-1} \mathrm{~cm}^{-1}$ at $785 \mathrm{~nm}$ in DW [22]. PPa is known to have a molar absorption coefficient of $9.98 \times$ $10^{4} \mathrm{M}^{-1} \mathrm{~cm}^{-1}$ at $413 \mathrm{~nm}$ in DMF [23]. These values were used to calculate the average number of MMP2P conjugated per GNR in the MMP2P-GNR conjugates.

\section{Analysis of fluorescence and singlet oxygen gen- eration (SOG) from the MMP2P-GNR conjugate}

To observe fluorescence quenching characteristics, the MMP2P-GNR conjugates were dissolved in phosphate-buffered saline ([PBS], $6.7 \mathrm{mM}$; pH 7.4; $\mathrm{NaCl}, 154 \mathrm{mM}$ ), and their fluorescence spectra were measured (Ex. $410 \mathrm{~nm}$ ). In this study, a concentration equivalent to $1 \mu \mathrm{M}$ PPa was used for each solution. For comparison, free PPa was dissolved in PBS containing $1 \%(\mathrm{v} / \mathrm{v})$ Tween 20 to prevent fluorescence quenching due to aggregation (Supplementary Material: Figure S4A). 
To observe an inhibitory characteristic with respect to SOG, both singlet oxygen sensor green and the MMP2P-GNR conjugate were dissolved in PBS solution saturated with oxygen gas. For the measurement of SOG from free $\mathrm{PPa}$, both singlet oxygen sensor green and PPa were dissolved in PBS containing $1 \%(\mathrm{v} / \mathrm{v})$ Tween 20 . The final concentration of free PPa and MMP2P-GNR in the aqueous solutions was equivalent to $1 \mu \mathrm{M}$ PPa. SOG from the MMP2P-GNR conjugate was quantified by comparing to that from free PPa. Each solution was irradiated with a CW laser beam at $670 \mathrm{~nm}$ (irradiation dose rate, $68 \mathrm{~mW} \mathrm{\textrm {cm } ^ { - 2 }}$ and irradiation dose, $2 \mathrm{~J} \mathrm{~cm}^{-2}$ ). All experiments were performed in triplicate.

\section{Dispersion stability of MMP2P-GNR}

To check the dispersion stability of MMP2P-GNR in the various aqueous solutions, MMP2P-GNRs were dispersed in each of PBS and a cell culture medium (Dulbecco's modified Eagle's medium without phenol red) containing $10 \%$ fetal bovine serum (FBS). The final concentration of GNRs in the solutions was adjusted to $0.5 \mathrm{nM}$. The solutions were maintained at $25^{\circ} \mathrm{C}$ for $24 \mathrm{~h}$. UV/Vis spectra of the sample solutions were taken after $10 \mathrm{~min}$ and $24 \mathrm{~h}$ of incubation at $25^{\circ} \mathrm{C}$

\section{In vitro cell study for fluorescence activation of MMP2P-GNR}

MMP2-positive HT1080 cells and MMP2-negative BT20 cells in culture media were plated onto a LabTek II Chambered Coverglass (Nalge Nunc International Corp.) at a density of 90,000 cells/well, and incubated for $24 \mathrm{~h}$ to allow for cell attachment. Thereafter, MMP2P-GNR solution was diluted with culture medium to achieve a concentration equivalent to $5 \mu \mathrm{M} \mathrm{PPa}$, and added at 600 $\mu \mathrm{L}$ per well. The cells were incubated at $37^{\circ} \mathrm{C}$ for $15 \mathrm{~h}$ after which the culture medium was removed, and the cells were washed 3 times with fresh culture medium. Fluorescence images (Ex. $405 \mathrm{~nm}$ and Em. 646-753 $\mathrm{nm})$ were acquired using a confocal laser scanning microscope (ZEISS LSM 510 META).

\section{In vitro phototoxicity test}

For in vitro phototoxicity tests, HT1080 and BT20 cells were seeded in 96-well plates at a density of 9,000 cells/well. The next day, the photosensitizer-oligopeptide conjugate (MMP2P) and the photosensitizer- gold nanorod conjugate (MMP2P-GNR) were diluted in cell culture medium to obtain a concentration equivalent to $5 \mu \mathrm{M} \mathrm{PPa}$. The existing culture medium was replaced with $200 \mu \mathrm{L}$ of fresh medium containing MMP2P or the MMP2P-GNR conjugate, and the cells were incubated for $15 \mathrm{~h}$. Mean- while, for the untreated control group, the same volume of a fresh culture medium with no photosensitizer included was added to a plate and incubated for $15 \mathrm{~h}$. Thereafter, the cells were washed twice with cell culture medium, and for the PDT group, light irradiation was carried out with a 670-nm CW laser beam at a dose of $10 \mathrm{~J} / \mathrm{cm}^{2}$ and at a dose rate of 40 $\mathrm{mW} / \mathrm{cm}^{2}$. After $24 \mathrm{~h}$, cell viability was measured by MTT analysis. Cell viability was calculated as a percentage compared to untreated control cells. Dark toxicity of the MMP2P and the MMP2P-GNR conjugate was also evaluated by incubating these compounds for $15 \mathrm{~h}$ at the same concentration without light treatment.

For the morphological analysis following light illumination, HT1080 and BT20 cells in culture media were plated onto a LabTek II Chambered Coverglass (Nalge Nunc International Corp.) at a density of 90,000 cells/well, and incubated for $24 \mathrm{~h}$ to allow for cell attachment. Thereafter, MMP2P-GNR solution was diluted in culture medium to a concentration equivalent to $5 \mu \mathrm{M} \mathrm{PPa}$, and added at $600 \mu \mathrm{L}$ per well. The cells were incubated at $37^{\circ} \mathrm{C}$ for $15 \mathrm{~h}$. Culture medium was then removed and the cells were washed 3 times with a fresh culture medium before receiving light treatment with a $670-\mathrm{nm} \mathrm{CW}$ laser beam at a dose of $10 \mathrm{~J} / \mathrm{cm}^{2}$ and at a dose rate of $40 \mathrm{~mW} / \mathrm{cm}^{2}$. Fluorescence and transmittance images were obtained using a confocal laser scanning microscope (Ex. 405 $\mathrm{nm}$ and Em. 646-753 nm) immediately following PDT.

\section{Results \& Discussion}

A transmission electron microscopy (TEM) image of the CTAB-coated GNR showed that the average length and width was $33.6 \pm 3.7$ and $8.7 \pm 0.9 \mathrm{~nm}$, respectively (aspect ratio, 3.9:1) (Figure 2A). From the analysis of the UV/Vis absorption spectrum, the maximum absorption wavelength of the CTAB-coated GNR is about $766 \mathrm{~nm}$, and a strong plasmon resonance spectrum was observed in the fluorescence wavelength of PPa, i.e., 650 to $780 \mathrm{~nm}$ (Figure 2B). The $\mathrm{UV} / \mathrm{Vis}$ absorption spectrum of the MMP2P-GNR in PBS solution shows additional absorption peaks at $387 \mathrm{~nm}$ (Soret band) and $670 \mathrm{~nm}$ (Q band), which represent absorption peaks by the photosensitizer PPa. Blue shift and broadening of the Soret band of PPa indicates that there was an aggregation between the conjugated photosensitizers. When the MMP2P-GNR was dissolved in DMF (Supplementary Material: Figure S5), narrow and clear peaks were shown at 413 and $670 \mathrm{~nm}$, and this is a typical feature of monomeric PPa. The molar ratio of conjugated MMP2P per GNR was calculated to be 4,960:1 from the UV/Vis spectrums of MMP2P-GNR in PBS and 


\section{DMF solutions.}

As mentioned above, the main plasmonic resonance band of the GNRs in the near-infrared region significantly overlapped with the emission band of PPa (Figure 2B). Therefore, we assumed that fluorescence and SOG of PPa may be inhibited due to energy transfer from the excited PPa to the GNRs and also partly due to self-quenching between the bound photosensitizers. Fluorescence spectra of the free $\mathrm{PPa}$ and MMP2P-GNR confirms the ultra-efficient quenching of the fluorescence in MMP2P-GNR compared with free PPa (Figure 2C). From the quantitative analysis of the fluorescence and SOG, the MMP2P-GNR conjugate emitted a fluorescence intensity corresponding to $0.4 \% \pm 0.01 \%$ and an SOG efficiency of $0.89 \% \pm 1.04 \%$ compared to free PPa (Figure 2D). This confirms that, when PPa is adjacent to the GNR surface, the fluorescent signal and SOG are effectively inhibited. In other words, the fluorescence and SOG of PPa recover when the compound is released from the GNR surface.

To check the dispersion stability, MMP2P-GNR was dispersed in each of PBS solution and cell culture medium containing $10 \%$ fetal bovine serum (FBS). The
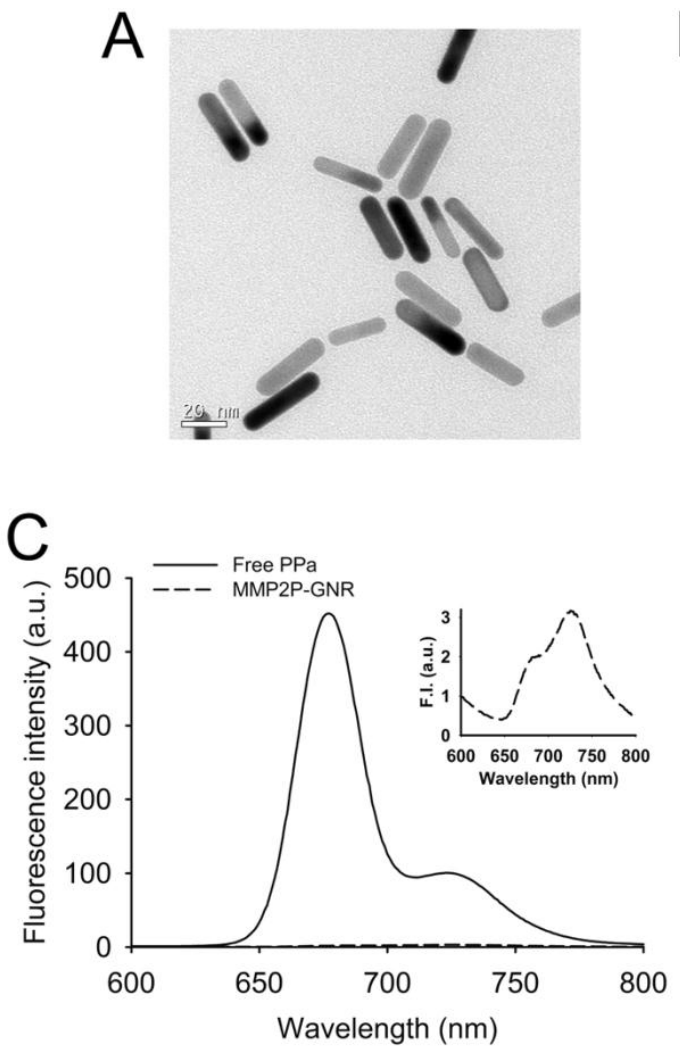

Figure 2. Development and characterization of MMP2P-GNR. (A) Transmission electron microscopy (TEM) image of CTAB-coated GNRs. (B) UV/Vis absorption spectrum of the CTAB-coated GNR and MMP2P-GNR. (C) Fluorescence spectra of free PPa and MMP2P-GNR (Ex. $410 \mathrm{~nm}$ and Em. 600-800 nm). Box: fluorescence spectrum of MMP2P-GNR conjugate. (D) Relevant fluorescence intensity (left: Ex. $410 \mathrm{~nm}$ and Em. $677 \mathrm{~nm}$ ) and SOG (right) of I $\mu$ M of the MMP2P-GNR conjugate. (The measured fluorescence and SOG of I $\mu \mathrm{M}$ PPa was converted to $100 \%)$.
$\mathrm{UV} / \mathrm{Vis}$ spectra of all the test solutions were taken after $10 \mathrm{~min}$ and $24 \mathrm{~h}$ of incubation at $25^{\circ} \mathrm{C}$ (Supplementary Material: Figure S6). The UV/Vis spectra of all the test solutions did not change for $24 \mathrm{~h}$, indicating that there was no aggregation or precipitation of GNRs.

We then evaluated protease-mediated activation of fluorescence and SOG in vitro using HT1080 cells (human fibrosarcoma cell line), which overexpress MMP2, and BT20 cells (human mammary adenocarcinoma cell line), which lack detectable expression of MMP2. Figure 3 shows fluorescence in both cell lines treated with MMP2P-GNR. The cancer cell line HT1080 emitted a much stronger fluorescence compared to BT20 cells. This result suggests that in the HT1080 cells, the peptide substrate of the MMP2P-GNR conjugate was cleaved by the MMP2, separating the photosensitizer from the GNR, and thereby facilitating the emission of fluorescent signal. We can speculate that if the photosensitizer-GNR conjugate were to be administered to a patient, detection of fluorescence following light radiation would accurately identify tissues in which MMP2 was overexpressed.

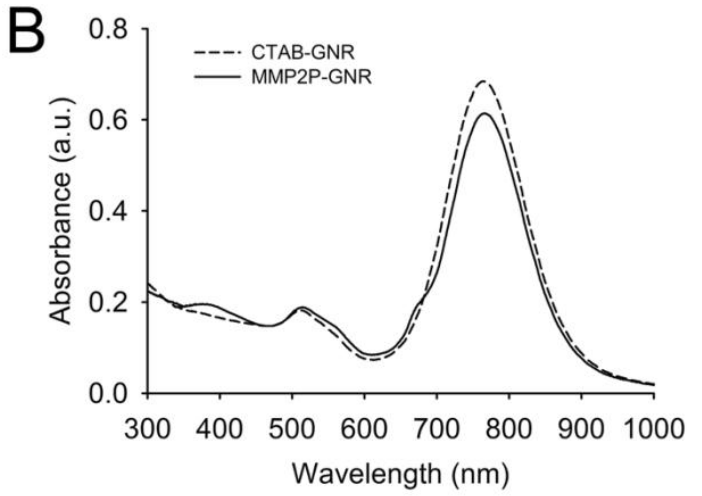

D

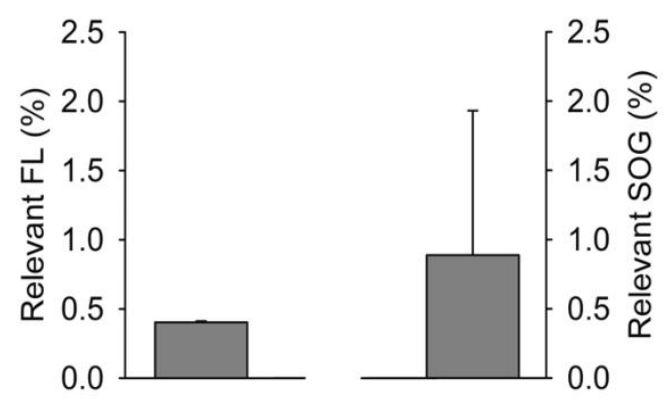


When the cell lines were treated with MMP2P and when PDT was performed, both HT1080 and BT20 exhibited low cell viabilities representing high phototoxicity (Figure 4). However, when the cells were treated with MMP2P-GNR followed by PDT, HT1080 exhibited a cell viability of $16 \%$, whereas the cell line BT20 exhibited a cell viability of $63 \%$. This supports the theory that the phototoxicity induced by the MMP2P-GNRs was the result of selective activation by the MMP2 present in the HT1080 cell line. Since it is generally believed that nano-sized drug carriers accumulate at tumor sites by the so-called enhanced permeation and retention effect [24], an improved outcome of selective PDT in vivo is expected. No dark toxicities were observed in both cell lines when treated with either MMP2P or MMP2P-GNR.

Blebbing of the cell membrane of HT1080 cells was observed upon light illumination whereas no apparent change in the cell morphologies was shown in the BT20 cells (Figure 5). This is likely a result of the ROS generated from photosensitizers that have been activated by the MMP2 present in the HT1080 cell line, and this subsequently leads to the observed

Figure 3. Confocal microscopy images of unfixed HTI080 and BT20 cells treated with MMP2P-GNR (5 $\mu$ M $\mathrm{PPa}$ eq.). Top row: fluorescence images of the cells (Ex. $405 \mathrm{~nm}$ and Em. 646-753 nm). The fluorescence signals are from PPa. Bottom row: transmitted light images merged with the fluorescence image above (Magnification: $80 \times)$.

disruption of the cell membrane.

Fluorescence and SOG were efficiently inhibited by conjugating photosensitizers onto gold nanorods via a protease-cleavable peptide linker. MMP2P-GNRs demonstrated selective activation in near-infrared fluorescence and phototoxicity in cells in which MMP2 is overexpressed. This novel design of an enzyme-activatable PDT agent may be useful in near-infrared fluorescence imaging and in subsequent PDT of various disease sites in which a specific protease is overexpressed.
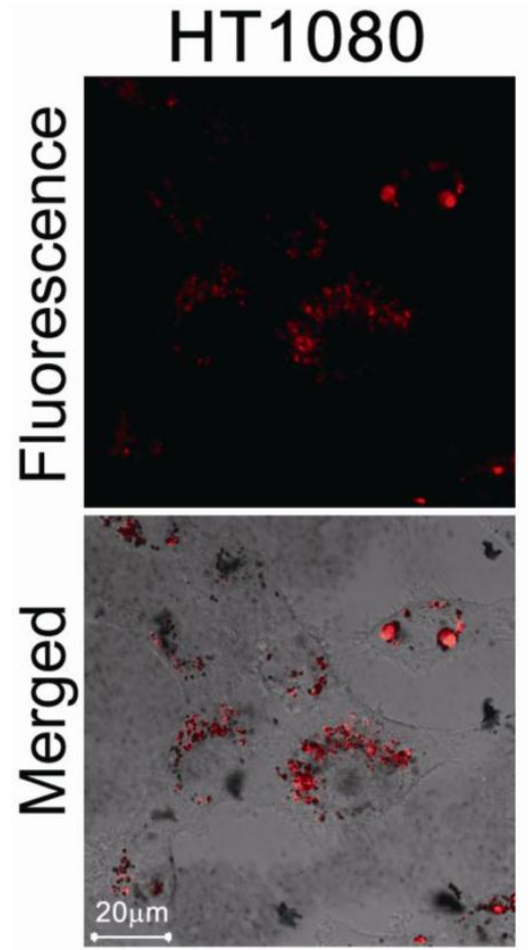
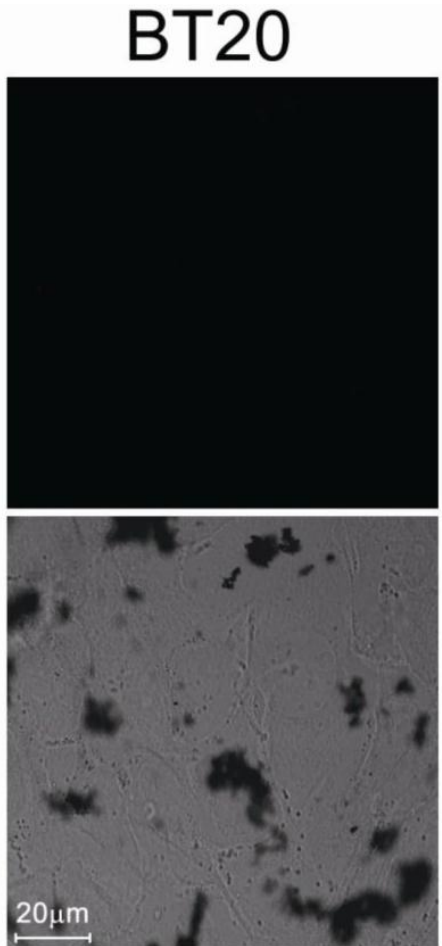
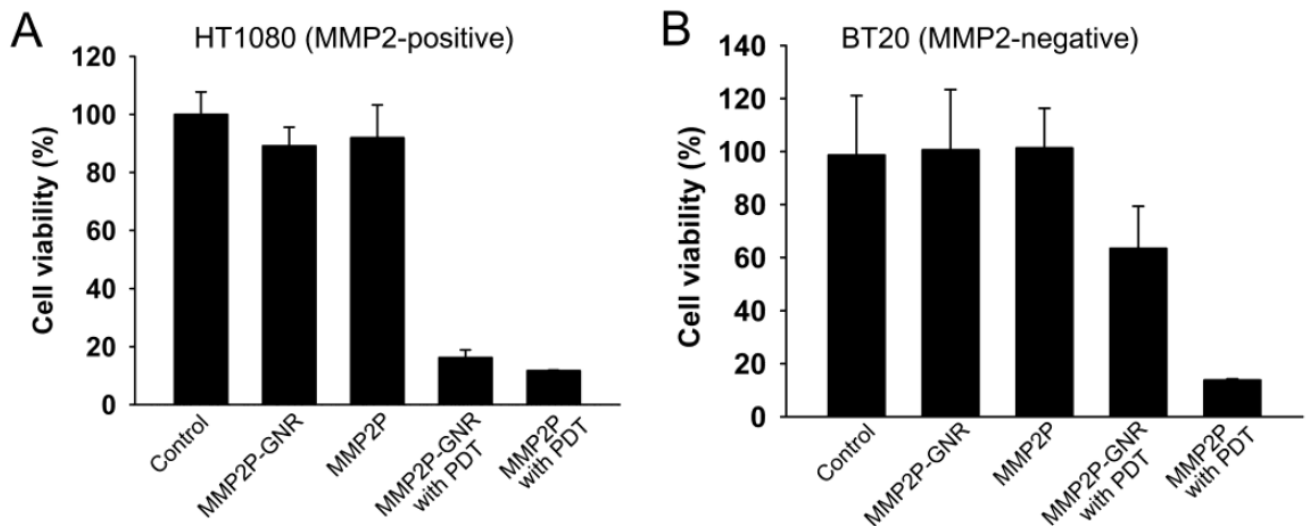

Figure 4. In vitro photodynamic efficacy of MMP2P and MMP2P-GNR. (a) HTI080 (MMP2-positive) and (b) BT20 (MMP2-negative) cells were treated with either $5 \mu \mathrm{M}$ PPa equivalent MMP2P or MMP2P-GNR for $15 \mathrm{~h}$ in the dark prior to light illumination with 670-nm diode laser. Cell viability was determined by MTT assay. Data represent mean \pm S.D. $(n=4)$. 


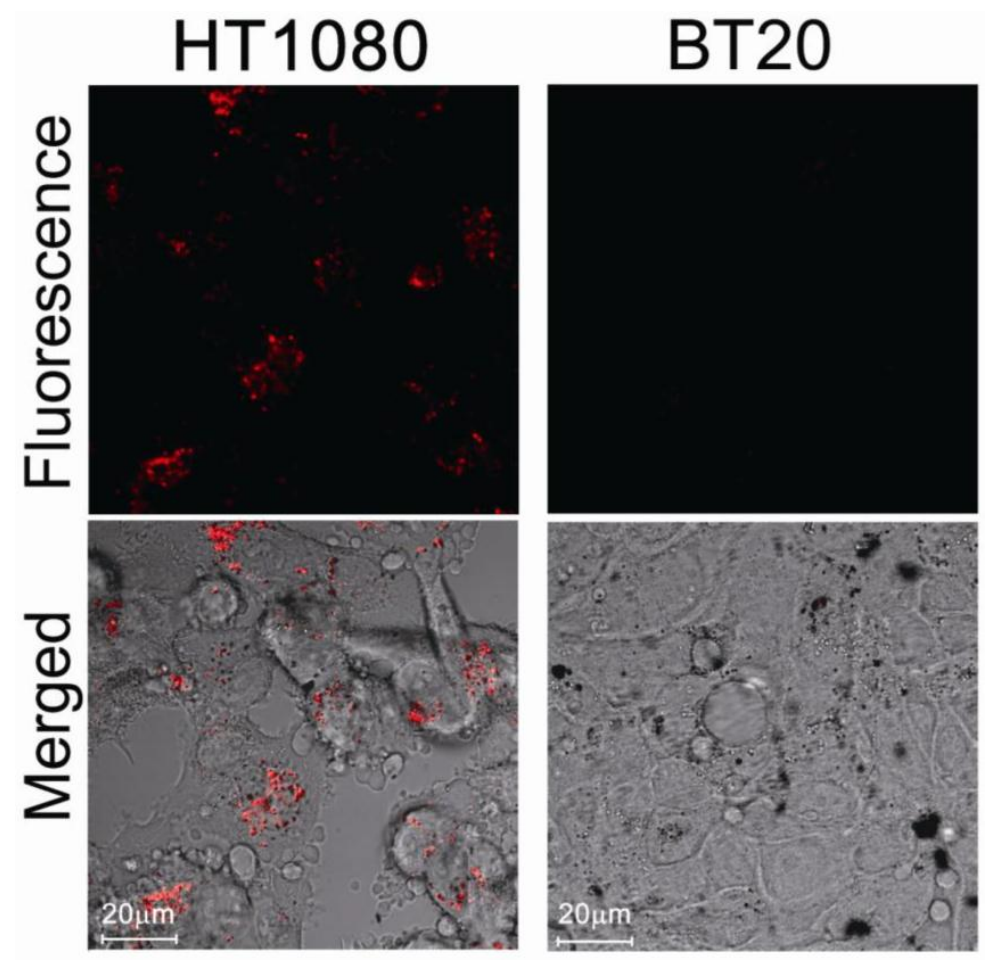

Figure 5. Confocal microscopy images of unfixed HTI080 and BT20 cells treated with MMP2P-GNR (5 $\mu$ M PPa eq.) followed by light illumination (light dose, $10 \mathrm{~J} / \mathrm{cm}^{2}$ and light dose rate, $40 \mathrm{~mW} / \mathrm{cm}^{2}$ ). Top row: fluorescence images of the cells (Ex. $405 \mathrm{~nm}$ and Em. 646-753 $\mathrm{nm}$ ). The fluorescence signals are from PPa. Bottom row: transmitted light images merged with the fluorescence image above (Magnification, $80 \times)$.

\section{Supplementary Material}

Fig.SI-S6. http://www.thno.org/v02p0190sI.pdf

\section{Acknowledgements}

This work was supported by a National Cancer Center grant from the Republic of Korea (1010150-2), and the Fundamental R\&D Program for Core Technology of Materials by the Ministry of Knowledge Economy, Republic of Korea.

\section{Conflict of Interest}

The authors have declared that no conflict of interest exists.

\section{References}

1. Hopper C. Photodynamic therapy: a clinical reality in the treatment of cancer. Lancet Oncol. 2000; 1: 212-9.

2. Dolmans DE, Fukumura D, Jain RK. Photodynamic therapy for cancer. Nat Rev Cancer. 2003; 3: 380-7.

3. Choi $\mathrm{Y}$, Weissleder R, Tung $\mathrm{CH}$. Selective antitumor effect of novel protease-mediated photodynamic agent. Cancer Res. 2006; 66: 7225-9.

4. Zheng G, Chen J, Stefflova K, Jarvi M, Li H, Wilson BC. Photodynamic molecular beacon as an activatable photosensitizer based on protease-controlled singlet oxygen quenching and activation. Proc Natl Acad Sci U S A. 2007; 104: 8989-94.

5. Gabriel D, Campo MA, Gurny R, Lange N. Tailoring protease-sensitive photodynamic agents to specific disease-associated enzymes. Bioconjug Chem. 2007; 18: 1070-7.

6. Bae BC, Na K. Self-quenching polysaccharide-based nanogels of pullulan/folate-photosensitizer conjugates for photodynamic therapy. Biomaterials. 2010; 31: 6325-35.

7. Koo H, Lee H, Lee S, Min KH, Kim MS, Lee DS, et al. In vivo tumor diagnosis and photodynamic therapy via tumoral pH-responsive polymeric micelles. Chem Commun (Camb). 2010; 46: 5668-70.

8. Park SY, Baik HJ, Oh YT, Oh KT, Youn YS, Lee ES. A smart polysaccharide/drug conjugate for photodynamic therapy. Angew Chem Int Ed Engl. 2011; 50: 1644-7.

9. Lee SJ, Koo H, Lee D-E, Min S, Lee S, Chen X, et al. Tumor-homing photosensitizer-conjugated glycol chitosan nanoparticles for synchronous photodynamic imaging and therapy based on cellular on/off system. Biomaterials. 2011; 32: 4021-9.

10. McCarthy JR, Weissleder R. Model systems for fluorescence and singlet oxygen quenching by metalloporphyrins. ChemMedChem. 2007; 2: 360-5.

11. Zhu Z, Tang Z, Phillips JA, Yang R, Wang H, Tan W. Regulation of singlet oxygen generation using single-walled carbon nanotubes. J Am Chem Soc. 2008; 130: 10856-7.

12. Jang B, Park JY, Tung CH, Kim IH, Choi Y. Gold nanorod-photosensitizer complex for near-infrared fluorescence imaging and photodynamic/photothermal therapy in vivo. ACS Nano. 2011; 5: 1086-94. 
13. Dulkeith E, Ringler M, Klar TA, Feldmann J, Munoz Javier A, Parak WJ. Gold nanoparticles quench fluorescence by phase induced radiative rate suppression. Nano Lett. 2005; 5: 585-9.

14. Jain PK, Lee KS, El-Sayed IH, El-Sayed MA. Calculated absorption and scattering properties of gold nanoparticles of different size, shape, and composition: applications in biological imaging and biomedicine. J Phys Chem B. 2006; 110: 7238-48.

15. Griffin J, Singh AK, Senapati D, Rhodes P, Mitchell K, Robinson $\mathrm{B}$, et al. Size- and distance-dependent nanoparticle surface-energy transfer (NSET) method for selective sensing of hepatitis C virus RNA. Chemistry. 2009; 15: 342-51.

16. Bremer $\mathrm{C}$, Tung $\mathrm{CH}$, Weissleder $\mathrm{R}$. In vivo molecular target assessment of matrix metalloproteinase inhibition. Nat Med. 2001; 7: 743-8.

17. Stearns ME, Wang M. Type IV collagenase (M(r) 72,000) expression in human prostate: benign and malignant tissue. Cancer Res. 1993; 53: 878-83.

18. Davies B, Waxman J, Wasan H, Abel P, Williams G, Krausz T, et al. Levels of matrix metalloproteases in bladder cancer correlate with tumor grade and invasion. Cancer Res. 1993; 53: 5365-9.

19. Zucker S, Hymowitz M, Conner C, Zarrabi HM, Hurewitz AN, Matrisian L, et al. Measurement of matrix metalloproteinases and tissue inhibitors of metalloproteinases in blood and tissues. Clinical and experimental applications. Ann N Y Acad Sci. 1999; 878: 212-27.

20. Fang J, Shing Y, Wiederschain D, Yan L, Butterfield C, Jackson $\mathrm{G}$, et al. Matrix metalloproteinase- 2 is required for the switch to the angiogenic phenotype in a tumor model. Proc Natl Acad Sci US A. 2000; 97: 3884-9.

21. Liao H, Hafner JH. Gold Nanorod Bioconjugates. Chemistry of Materials. 2005; 17: 4636-41.

22. Orendorff CJ, Murphy CJ. Quantitation of metal content in the silver-assisted growth of gold nanorods. J Phys Chem B. 2006; 110: 3990-4.

23. AL-OMARI S. Energy transfer of pyropheophorbide a methyl ester in dimethylformamide solutions. Rom J Biophys. 2010; 20: 295-314.

24. Bechet D, Couleaud P, Frochot C, Viriot ML, Guillemin F, Barberi-Heyob M. Nanoparticles as vehicles for delivery of photodynamic therapy agents. Trends Biotechnol. 2008; 26: 612-21. 